\title{
Escasa difusión de frecuencias de transporte turístico y logística, en la ciudad de Riobamba, análisis legal, matemático - estadístico.
}

Legal, mathematical and statistical analyses regarding the lack of information on tourism transportation and logistics in Riobamba, Ecuador

María Fernanda Herrera Chico. ${ }^{1}$, Diego Arguello. ${ }^{2}$, Jenny Margoth Villamarín Padilla. ${ }^{3}$ \& Xavier Alejandro Guerra Sarche. ${ }^{4}$

Recibido: 10-03-2019 / Revisado: 15-03-209 /Aceptado: 04-04-2019/ Publicado: 13-05-2019

\begin{abstract}
.
DOI: https://doi.org/10.33262/cienciadigital.v3i2.2.463

The means of transport over time have favored notably with the progress and growth of the tourism sector because tourists seek to move easily from one place to another. This article analyzes elements of tourism activity such as transport and logistics, in order to improve the frequency-information system regarding tourist places of Riobamba and Chimborazo province whose capital is precisely this canton. The main aim of this project is presenting improve recommendations for the information diffusion related to means of transport in order to satisfy the constant growing tourism demand requirements. This research was developed using a descriptive and analytical analysis for the information collection, observation, study and result analyses. Into a worldwide tourism context, the cultural, educational, political, social, and financial exchanges is strongly influenced by the means of transport. Due to this fact, this research focuses on quantitatively determine whether the transport offer reach an appropriate level of satisfaction in Riobamba and Chimborazo province from a national and foreign tourism demand perspective.
\end{abstract}

\footnotetext{
${ }^{1}$ Escuela Superior Politécnica del Chimborazo, Facultad de Administración de Empresas, Carrera de Gestión del Transporte, Riobamba, Ecuador, maria.herrerac@espoch.edu.ec

2 Escuela Superior Politécnica de Chimborazo, Facultad de Administración de Empresas, Carrera de Gestión del Transporte, Riobamba, Ecuador, diego.arguello@espoch.edu.ec

${ }^{3}$ Escuela Superior Politécnica de Chimborazo, Facultad de Administración de Empresas, Carrera de Gestión del Transporte, Riobamba, Ecuador, j_villamarin@espoch.edu.ec

${ }^{4}$ Escuela Superior Politécnica del Chimborazo, Facultad de Administración de Empresas, Carrera de Gestión del Transporte, Riobamba, Ecuador, xavier.guerra@espoch.edu.ec
} 
Keywords: Riobamba, tourism, transport, information system, local development.

\section{Resumen.}

Los medios de transporte a lo largo del tiempo han favorecido notablemente con el progreso y crecimiento del sector turístico, debido a que los turistas buscan desplazarse de manera fácil de un lugar a otro. El presente artículo analiza elementos de la actividad turística y la importancia del transporte y su logística, para la difusión de frecuencias provinciales a los principales lugares turísticos del cantón Riobamba y de la provincia de Chimborazo. Se realiza un estudio de la importancia de mejorar la difusión de los medios de transporte con la finalidad de satisfacer la demanda turística, la cual crece constantemente. La investigación se desarrolló con un análisis descriptivo y analítico, recolecta información, observa, estudia y relaciona los hechos y resultados obtenidos. En el mundo turístico, los intercambios culturales, educativos, políticos, sociales, financieros, se producen gracias a los medios de transporte, en tal sentido el trabajo investigativo e enfoca a determinar de manera cuantitativa el número de turistas tanto nacionales como extranjeros que visitan la ciudad de Riobamba y la provincia de Chimborazo a fin de relacionar los mismos con la utilización de los medios de transporte que se oferta, de lo cual se espera determinar si dichos medios están acordes con la demanda de los turistas.

Palabras claves: Riobamba, turismo, transporte, difusión, desarrollo local.

\section{Introducción.}

El turismo es una de las actividades económicas con mayor crecimiento, su importancia se evidencia a través de la generación de ingresos en la mayoría de países (1). El turismo integra diversas actividades humanas como son: transporte, hospedaje, diversión, cultura, con diversos propósitos acorde a los múltiples deseos y motivaciones humanas (2), en relación a lo mencionado, el realizar una investigación sobre el turismo y el transporte en sus diferentes modalidades es de imperiosa necesidad por ser un importante instrumento para el desarrollo económico de los pueblos.

El sector del turismo es una fuente de ingresos en permanente crecimiento por su expansión y diversificación, generando ingresos por: exportaciones, generación de empleo y constante incremento de la inversión, tanto interna como externa en infraestructura, esto hace que el trabajo se enfoque en primer lugar en realizar un análisis estadístico del número de turistas que visitan la ciudad de Riobamba para entender el efecto del turismo en el sector, porque según las proyecciones a largo plazo de la Organización Mundial de Turismo (OMT) que se encuentran en el Tourism Towards 2030, la llegadas de turistas internacionales han registrado un crecimiento prácticamente ininterrumpido desde los 25 millones en 1950 hasta los 278 millones en 1980, los 528 millones en 1995 y los 1.035 millones en 2012. Así mismo, las previsiones a largo plazo de la OMT, indican que las llegadas de turistas internacionales crecerán un 3,3\% anualmente entre 2010 y 2030 hasta alcanzar los 1.800 millones en 2030. (3). 
La curiosidad humana, la necesidad de descanso, la emulación social, el alto nivel de renta y varios motivos más han dado lugar al fenómeno turístico. El número de viajeros crece y crece sin cesar, la oferta de lugares bonitos, de buen clima, de buena mesa, aumenta cada día. Aprovecharlo solo ha sido posible por las enormes mejoras en las técnicas de transporte y de comunicaciones. Gracias a la aplicación de las técnicas logísticas, el turismo se ha hecho mucho más posible. (4)

Para el profesional que trabaja en el campo de la Logística el crecimiento del turismo representa un gran desafío al cual debe enfrentarse, debido a las características de los servicios turísticos que requieren de un flujo intenso - cada día más complejo- de personas, mercancías y bienes e información, conjuntamente con una gran exigencia de lograr la satisfacción del turista en toda la cadena de consumo. (5)

El Ecuador es uno de los países con mayor biodiversidad del planeta, en los últimos años ha mantenido un interesante crecimiento turístico debido a sus maravillas naturales (1). Riobamba, conocida como "La Sultana de los Andes", o la "Ciudad de las Primicias", se encuentra ubicada en la zona céntrica del Ecuador a una altura de 2.764 metros sobre el nivel del mar, cuyos atractivos turísticos han hecho de esta urbe el lugar de visita habitual tanto de turistas nacionales como extranjeros; debiendo considerar también que en esta ciudad se protagonizaron hechos de trascendental importancia en la vida socio política del Ecuador. Su centro histórico mantiene un estilo colonial, rodeado de edificaciones modernas creando una imagen arquitectónica diferente. (6).

El Gobierno Autónomo Descentralizado Municipal en el Plan Estratégico de Desarrollo Cantonal de la ciudad de Riobamba para el año 2020 (pp. 90) (6), desarrolló las actividades económicas de la ciudad, siendo estas las siguientes: sector comercial $53.57 \%$ (tiendas, negocios de alimentos, bazares, venta de ropa, almacenes de repuestos y ferreterías), servicios profesionales y otros $24,22 \%$, sector manufacturero $9,10 \%$, agropecuario $7,10 \%$, servicios de alimentación, diversión y hospedaje 6,01\%, en relación a lo mencionado, la actividad turística mantiene una pequeña participación dentro de las actividades económicas de la ciudad de Riobamba, a pesar de ello, llama mucho la atención que el sector de transporte no aparezca con ningún porcentaje de participación, pese a ser otra de las fuentes permanentes de desarrollo económico tanto cantonal, provincial y nacional.

La investigación aborda la problemática de la inadecuada difusión de las frecuencias de las operadoras de transporte en sus diferentes clases de conformidad con la clasificación establecida en el artículo 51 de la Ley Orgánica de Transporte Terrestre, Tránsito y Seguridad Vial (7), en concordancia con el artículo 63 de su Reglamento de aplicación (8), actividad que se encuentran directamente ligada al turismo, por lo que se hace necesario realizar una apropiada logística de las rutas y frecuencias por medio del uso las nuevas tecnologías de la información a fin de mantener y fomentar el turismo en la provincia de Chimborazo, de esta manera los visitantes tendrán un mejor acceso a todos los medios de transporte terrestre para realizar sus actividades con mayor eficiencia, contribuyendo de manera efectiva al desarrollo económico del sector.

El turismo y el transporte se encuentran íntimamente ligados, por lo que es importante conceptualizar a cada uno de ellos de manera independiente para poder determinar de mejor 
manera lo que es el transporte en el turismo; para iniciar tenemos que el turismo es: "El conjunto de relaciones y fenómenos que se originan del acto o hecho jurídicos, que lleva a efecto un individuo para emprender (acto) o al realizar un viaje (hecho) y obtener, su estancia legal en lugar distinto al de su radicación". (9). Estos actos y hechos jurídicos se han vuelto más comunes en los ciudadanos del mundo, y han contribuido al constante crecimiento del turismo a nivel global. Decimos que son jurídicos porque el turista sea de la nacionalidad que este ostente, al ingresar a nuestro país goza de los mismos derechos y garantías amparado en el artículo 416 de la Constitución de la República del Ecuador. (10)

Los medios de transporte representan un alto impacto para la sociedad, pues para realizar un desplazamiento en su gran mayoría se requiere de éstos; así también, en la actualidad se puede notar que detrás del progreso del transporte se encuentran los adelantos tecnológicos, los cuales han coadyuvado en la difusión y desarrollo de los medios de transportación, a través de: mejorar el factor tiempo, economía, confort y el beneficio de escoger y conocer el transporte y horario más adecuado para trasladarse. (11)

Este concepto va ligado al hecho de que para realizar turismo se hace necesaria la utilización de otros elementos sustanciales como lo expresa Donald Lundberg, quien manifiesta que el turismo "es el negocio del transporte, atención alimenticia y diversión del turista; sus componentes son muchos y variados". Son parte principal del turismo "la economía, los negocios, la ecología, la geografía, el gobierno y la legislación". (12)

En el contexto en el cual "El Turismo es un fenómeno social que consiste en el desplazamiento voluntario y temporal de individuos o grupos de personas que fundamentalmente por motivos de recreación, descanso, cultura o salud, se trasladan de un lugar de residencia habitual a otro en el que o ejercen alguna actividad lucrativa ni remunerada, generando múltiples interrelaciones de suma importancia económica y cultural" (13) y que uno del componente del sector turístico es el transporte. (14).

El profesional logístico no debe obviar que el mercado turístico es altamente competitivo, donde el valor añadido cobra singular fuerza a través de la experiencia única, cuestión esta que ha obligado a la actividad turística a ofrecer un servicio superior, no solamente en términos de calidad, sino también de creatividad y donde se le impone dejar de ser una economía de servicios para convertirse en una economía de experiencias. (4) (Cabeza, 2001)

Así Mismo, la tecno ciencia ha perfeccionado cada uno de los medios de transporte, es el que ha permitido mejorar el factor espacio - tiempo, economía, confort y la conveniencia de escoger el más adecuado para trasladarse. Lo trascendente de este avance tecnológicocientífico es que ha coadyuvado a relacionar a los seres humanos, a efectuar intercambios culturales, a pesar que saliendo de los límites de la estratosfera se puede mantener contacto con otros seres, sea esto especulativo o no. (11)

Las infocomunicaciones han revolucionado la Logística, permitiendo que muchos problemas encontrados sean resueltos, entre ellos: tener una información cierta y rápida sobre el nivel de empleo de muchos recursos turísticos en cualquier lugar del mundo, actualmente es posible reservar una plaza hotel en cualquier lugar de la Tierra con una gran 
rapidez, al igual que un billete de avión o de barco o de tren. Lo segundo, es que han permitido la resolución más rápida de muchos problemas matemáticos difíciles que necesitan de aproximaciones sucesivas y en los que solo mediante muchas repeticiones de un mismo procedimiento puede llegarse a una solución aceptable. Lo tercero, es la realización electrónica de muchas transacciones comerciales y su simplificación. Lo cuarto, la presentación de la oferta turística en páginas web, de contenido sugestivo y agradable. Lo quinto, seguir con precisión la situación de cualquier producto que se transporte en cualquier lugar del mundo. (4)

La combinación de ordenador y telecomunicaciones, hoy fundamental para la solución de los problemas logísticos, tiene en el caso del turismo una especial significación. Hoy, cualquier posible turista comienza por examinar en internet sus posibles destinos, comunica con sus hoteles o sus agencias de viajes por FAX o teléfono, puede contratar sus billetes empleando líneas ADSL y pagarlos empleando tarjetas de crédito. (4)

Las principales facilidades para los turistas dentro de los servicios que se ofertan en la provincia de Chimborazo son: Museo de la Ciudad, visita Iglesias y edificios Arquitectónicos de la ciudad. En lo referente al recorrido en Tren, la compra de boletos se lo puede realizar en la página web de Ferrocarriles del Ecuador, esta es una opción para ahorra tiempo a los turistas, a través de esta pueden consultar horarios, precio, ruta e incluso comprar los tickets.

La investigación plantea los siguientes ámbitos estratégicos, para la intervención y fortalecimiento de la difusión de frecuencias de transporte provincial dentro de la situación turística de la provincia de Chimborazo. La elaboración de las estrategias se basa en las áreas funcionales de PLADETUR 2020 (15) y en el plan estratégico institucional del GADM Riobamba 2017 (16).

En el Ecuador la movilidad de las personas extranjeras está garantizada como un principio, es decir de acuerdo al artículo 416 de la Constitución de la República que trata de las relaciones internacionales nuestro país propugna el principio de ciudadanía universal, la libre movilidad de todos los habitantes del planeta y el progresivo fin de la condición de extranjero como elemento transformador de las relaciones desiguales entre los países, especialmente Norte-Sur. Para garantizar este principio constitucional no únicamente para la libre movilidad de los extranjeros sino de también de los ecuatorianos, mediante Registro Oficial Suplemento 398 de 07 de agosto del 2008 se promulga la Ley Orgánica de Tránsito, Transporte Terrestre y Seguridad Vial (7), en la que en su artículo 51 determina las clases de servicio de transporte terrestre en Público, Comercial, Por cuenta propia, y Particular.

En lo que respecta al transporte terrestre público, la citada ley en su artículo 55 dispone que este se considera un servicio estratégico del Estado, así como la infraestructura y equipamiento auxiliar que se utilizan en la prestación del servicio. Antes de la promulgación de la ley, las rutas y frecuencias a nivel nacional pertenecías a cada una de las prestadoras del servicio, por lo que a fin de regular y controlar la piratería en el transporte en la actualidad las rutas y frecuencias son de propiedad exclusiva del Estado, las cuales podrán ser comercialmente explotadas mediante contratos de operación. 
De la misma manera, antes de la promulgación de esta ley el tránsito estuvo a cargo de la Policía Nacional a través de la Dirección Nacional de Tránsito, pero en cumplimiento a los principios constitucionales de descentralización y desconcentración administrativa se crea en primer lugar la Agencia Nacional de Regulación y Control del Transporte Terrestre, Tránsito y Seguridad Vial, es el ente encargado de la regulación, planificación y control del transporte terrestre, tránsito y seguridad vial en el territorio nacional, en el ámbito de sus competencias, con sujeción a las políticas emanadas del Ministerio del Sector, es decir este es un ente adscrito al Ministerio de Transporte y Obras Públicas, encargado exclusivamente del ámbito del transporte terrestre, el tránsito y la seguridad vial en todo el territorio ecuatoriano, y se les transfiere también estas competencias a los Gobiernos Autónomos Descentralizados Regionales y Municipales dentro del ámbito de sus circunscripciones territoriales para que sean estos quienes confieran los permisos y autorizaciones a las operadoras del transporte terrestre en las diferentes modalidades, las mismas que de acuerdo con el artículo 63 del Reglamento a la Ley de Transporte Terrestre, Tránsito y Seguridad Vial (8), se encuentran clasificados dentro de su clase y ámbito de aplicación de la manera que de manera textual se transcribe a continuación.

Art. 63.- Los servicios de transporte terrestre de acuerdo a su clase, tipo y ámbito podrán prestarse en los siguientes vehículos, cuyas características se establecerán en la reglamentación y normas INEN vigentes:

Tabla 1. Servicios de transporte terrestre de acuerdo a su clase, tipo y ámbito de operación.

\begin{tabular}{|c|c|c|c|c|c|}
\hline \multirow{5}{*}{1} & \multirow{5}{*}{$\begin{array}{c}\text { Transporte } \\
\text { terrestre } \\
\text { público }\end{array}$} & \multirow[t]{2}{*}{1.1} & \multirow{2}{*}{$\begin{array}{l}\text { Transporte } \\
\text { Intracantonal }\end{array}$} & a & $\begin{array}{l}\text { Transporte Colectivo: Buses y minibuses. Los mismos que pueden } \\
\text { ser convencionales, de entrada baja o piso bajo. }\end{array}$ \\
\hline & & & & b & $\begin{array}{l}\text { Transporte Masivo: Tranvías, monorriel, metros, trolebuses, buses } \\
\text { articulados y buses biarticulados. }\end{array}$ \\
\hline & & 1.2 & $\begin{array}{l}\text { Transporte } \\
\text { Interprovincial }\end{array}$ & & Buses y minibuses y buses tipo costa. \\
\hline & & 1.3 & $\begin{array}{c}\text { Transporte } \\
\text { Intrarregional e } \\
\text { Interprovincial }\end{array}$ & & Buses y minibuses, microbuses y buses tipo costa. \\
\hline & & 1.4 & $\begin{array}{c}\text { Transporte } \\
\text { Internacional y } \\
\text { Fronterizo }\end{array}$ & & Buses. \\
\hline 2 & $\begin{array}{c}\text { Transporte } \\
\text { terrestre } \\
\text { comercial }\end{array}$ & 2.1 & $\begin{array}{l}\text { Transporte } \\
\text { Intracantonal }\end{array}$ & $\mathrm{a}$ & $\begin{array}{l}\text { Transporte Escolar e Institucional: Furgonetas, microbuses, mini } \\
\text { buses y buses }\end{array}$ \\
\hline
\end{tabular}




\begin{tabular}{|c|c|c|c|c|c|c|c|c|}
\hline & & & & & & b1 & Convencional & $\begin{array}{l}\text { Automóvil de } 5 \text { pasajeros, } \\
\text { incluido el conductor. }\end{array}$ \\
\hline & & & & & & b2 & Ejecutivo & $\begin{array}{l}\text { Automóvil de hasta } 5 \\
\text { pasajeros, incluido el } \\
\text { conductor. }\end{array}$ \\
\hline & & & & $\mathrm{c}$ & $\begin{array}{c}\text { Servicio } \\
\text { alternativo- } \\
\text { excepcional }\end{array}$ & \multicolumn{3}{|c|}{$\begin{array}{l}\text { Tricimotos, moto taxis, triciclos motorizados } \\
\text { (vehículos de tres ruedas). }\end{array}$} \\
\hline & & & & d & Carga liviana & \multicolumn{3}{|c|}{$\begin{array}{l}\text { Vehículos tipo camioneta de cabina sencilla con } \\
\text { capacidad de carga de hasta } 3.5 \text { toneladas. } \\
\text { toneladas }\end{array}$} \\
\hline & & & & $\mathrm{e}$ & Carga pesada & \multicolumn{3}{|c|}{$\begin{array}{l}\text { Vehículos y sus unidades de carga, con capacidad de } \\
\text { carga de más de } 3.5 \text { toneladas. } \\
\text { toneladas. }\end{array}$} \\
\hline & & & & $\mathrm{f}$ & Fronterizo & \multicolumn{3}{|c|}{$\begin{array}{l}\text { El mismo que se regulará por los acuerdos } \\
\text { internacionales vigentes. }\end{array}$} \\
\hline & & & & a & $\begin{array}{c}\text { Transporte } \\
\text { escolar e } \\
\text { institucional } \\
\end{array}$ & \multicolumn{3}{|c|}{ Furgonetas, microbuses, mini buses y buses. } \\
\hline & & & & $\mathrm{b}$ & Turismo & \multicolumn{3}{|c|}{$\begin{array}{l}\text { Vehículos todo terreno livianos, furgonetas, } \\
\text { microbuses, mini buses y buses. }\end{array}$} \\
\hline & & & Transporte & $\mathrm{c}$ & Carga liviana & \multicolumn{3}{|c|}{$\begin{array}{l}\text { Vehículos con capacidad de carga de hasta } 3.5 \\
\text { toneladas. }\end{array}$} \\
\hline & & & Interprovincial & d & $\begin{array}{l}\text { Transporte } \\
\text { mixto }\end{array}$ & \multicolumn{3}{|c|}{$\begin{array}{c}\text { Vehículos con capacidad de carga de hasta } 1.2 \\
\text { toneladas y hasta } 5 \text { pasajeros incluido el conductor. } \\
\text { incluido el conductor. }\end{array}$} \\
\hline & & & & $\mathrm{e}$ & Carga pesada & \multicolumn{3}{|c|}{$\begin{array}{c}\text { Vehículos y sus unidades de carga con capacidad de } \\
\text { carga de más de } 3.5 \\
\text { toneladas. }\end{array}$} \\
\hline & & & & a & Turismo & \multicolumn{3}{|c|}{$\begin{array}{l}\text { Vehículos todo terreno livianos, furgonetas, mini } \\
\text { buses y buses. }\end{array}$} \\
\hline & & & Interprovincial & $\mathrm{b}$ & Carga pesada & \multicolumn{3}{|c|}{$\begin{array}{l}\text { Vehículos de carga con peso bruto vehicular } \\
\text { superior a } 3.5 \text { toneladas, y unidades de carga. }\end{array}$} \\
\hline & & & & $\mathrm{c}$ & Pasajeros & \multicolumn{3}{|c|}{ Buses } \\
\hline & & & & a & \multicolumn{4}{|c|}{ Buses, mini buses, furgonetas, vehículos livianos. } \\
\hline & $\begin{array}{c}\text { 1ransporte } \\
\text { terrestre }\end{array}$ & & intracantonal, & $\mathrm{b}$ & \multicolumn{4}{|c|}{ Vehículos con capacidad de carga de hasta 3.5 toneladas. } \\
\hline & $\begin{array}{l}\text { por cuenta } \\
\text { propia }\end{array}$ & & $\begin{array}{l}\text { intrarregional, } \\
\text { interprovincial }\end{array}$ & $\mathrm{c}$ & \multicolumn{4}{|c|}{$\begin{array}{l}\text { Vehículos y sus unidades de carga con capacidad de carga de más de } \\
\qquad 3.5 \text { toneladas. }\end{array}$} \\
\hline
\end{tabular}

Fuente: Elaboración propia. 
Con estos datos, se procedió a consultar a los encuestados el tipo de transporte que utilizan para su movilización hacia la provincia de Chimborazo y sus cantones, obteniendo como resultado que del total de turistas nacionales el $45 \%$ vino en auto propio, el $29 \%$ a través de cooperativas de transporte, el $26 \%$ furgonetas y servicio puerta a puerta. Los turistas extranjeros utilizaron en un $79 \%$ cooperativas de transporte, el $19 \%$ furgonetas y servicio puerta a puerta y únicamente el restante $2 \%$ se movilizó en un auto rentado. De aquí la importancia en recordar el criterio acertado de Ninoska Viloria (11) quien manifiesta que: La sinergia de los distintos medios de transporte provincial es un elemento clave para la captación de turistas. Los medios de transporte complementan esta actividad y son un factor determinante para el progreso de la ciudad, por ello es importante capacitar a dicho sector para que cumplan con las exigencias turísticas y logren satisfacer las necesidades y expectativas de los viajeros a través de: calidad en el servicio, seguridad y confort. (11)

Si nuestro país y especialmente la provincia de Chimborazo y el cantón Riobamba, se encuentran debidamente organizados con estos dos elementos importantes que generan desarrollo económico a través del turismo, es oportuno preguntarse qué se ha hecho desde el ámbito público y privado para que esta actividad se desenvuelva en su totalidad, determinándose que la principal actividad turística que se está coordinando y desarrollando es la capacitación, cuyo objetivo es complementar la cadena de valor turística, desarrollar actividades que sean integrales. Por ello, se realizan capacitaciones al sector turístico en bebidas y alimentos, operaciones turísticas, temas de recreación y áreas operativas: administradores de establecimientos, seguridad alimentaria, atención al cliente. En general, se trabaja para que en la provincia de Chimborazo y especialmente en la ciudad de Riobamba se pueda desarrollar un turismo que sea atractivo; sin embargo, es necesario hacer notar que dentro de estas actividades no se ha contado con el transporte como eje importante dentro de esta cadena de valor.

El transporte históricamente ha sido un pilar fundamental dentro de la actividad turística, debido a que en la mayoría de ocasiones es indispensable para el desplazamiento de los turistas desde un punto de origen hacia los diferentes destinos. Así también, es importante considerar que el sector turístico es una de las actividades con mayor crecimiento y demanda a nivel mundial, por tanto, el perfeccionamiento de los medios de transporte dentro de la ciudad de Riobamba, Provincia de Chimborazo, es esencial para el buen desenvolvimiento de las actividades turísticas, la optimización de tiempo y el confort. Considerando que el sector turístico forma parte del desarrollo económico de la provincia, es importante la vinculación de todos los actores inmersos en esta importante actividad para la mejor provisión de bienes y servicios turísticos.

\section{Metodologia.}

\section{Tipo y diseño de investigación}

Estudio transversal, descriptivo y analítico, en el cual se recolecta información de una muestra dada de elementos de la población una sola vez, de manera aleatoria (Malhotra, 2004). Se miden características durante un tiempo determinado. Además, se estudia y correlaciona los hechos. 


\section{Los métodos aplicados en la presente investigación son:}

Inductivo. - la presente investigación parte de antecedentes y teoría del turismo.

Revisión bibliográfica. - Información que proviene de fuentes gubernamentales, investigaciones relacionadas al tema de estudio, revisión bibliográfica con la finalidad de identificar y definir el problema, y a la vez responder a preguntas de investigación. (Malhotra, 2004).

Analítico descriptivo. - Describe características de la población objetivo a través de encuestas y entrevistas exhaustivas (Malhotra, 2004).

\section{Alcance de la investigación}

Descriptivo. - suministra información a través de encuestas, diseñada para obtener información específica de variables (Malhotra, 2004).

\section{Población de estudio}

- Turistas Nacionales e Internacionales.

- Informantes Calificados del sector turístico, responsables de atractivos turísticos culturales y gestores culturales.

\section{Unidad de análisis}

- 321 Turistas nacionales y 62 extranjeros que visitan la ciudad de Riobamba, con quienes se levantará información sobre las características principales y preferencias de las personas que visitan la ciudad.

\section{Resultados.}

Para tener una primera visión del problema, se realizó un primer la recopilación estadística por medio de encuestas realizadas a los turistas tanto nacionales como extranjero a fin de establecer su promedio de edad y género, este dato nos sirve para cuantificar el tipo de ingresos de cada población, además de la condición para poseer vehículo propio o al menos alquilado dependiendo cada caso; de este primer muestreo se obtuvo los que se muestran en la tabla 1, de un total de 382 turistas nacionales y extranjeros que visitaron la ciudad de Riobamba. 
Tabla 2. Género de turistas por rango de edad.

\begin{tabular}{cccc} 
Edad & Femenino & Masculino & Total \\
\hline $15-26$ & 40 & 47 & 87 \\
\hline $27-37$ & 83 & 66 & 149 \\
\hline $38-48$ & 26 & 45 & 71 \\
\hline $49-59$ & 25 & 22 & 47 \\
\hline $60-70$ & 14 & 11 & 25 \\
\hline$>71$ & 0 & 3 & 3 \\
\hline Total & 188 & 194 & 382
\end{tabular}

Fuente: Elaboración propia.

Del total de turistas encuestado, 321 son turistas nacionales con un total del $84 \%$ de encuestados y el restante $16 \%$ es decir 61 personas son turistas extranjeros, de los cuales se tiene un dato importante a través de la determinación de su nacionalidad de lo cual hemos obtenido los siguientes resultados detallados en la tabla 2.

Tabla 3. Procedencia de turistas extranjeros.

\begin{tabular}{lcc} 
Procedencia & Frecuencia & Porcentaje \\
\hline España & 14 & $23 \%$ \\
\hline Alemania & 9 & $15 \%$ \\
\hline Estados Unidos & 8 & $13 \%$ \\
\hline Francia & 7 & $11 \%$ \\
\hline Colombia & 7 & $11 \%$ \\
\hline Otros & 17 & $27 \%$ \\
\hline Total & 62 & $100 \%$
\end{tabular}

Fuente: Elaboración propia.

La ciudad de Riobamba y la provincia de Chimborazo tiene varios atractivos turísticos, dentro los cuales tenemos como principales el nevado Chimborazo, la ruta del tren y sus iglesias de tipo colonial, sin dejar otros atractivos turísticos como su gastronomía y sus parques, pero con fines de investigación se realizó un muestreo de los turistas con la finalidad de determinar el tipo de movilización que utilizan en sus desplazamientos se consultó sobre sus preferencias turísticas de lo cual nos arrojaron los siguientes resultados, conforme se indica en la tabla 3. 
Tabla 4. Preferencia de las actividades turísticas.

\begin{tabular}{|c|c|c|c|c|c|c|c|c|c|c|}
\hline \multirow{2}{*}{$\begin{array}{l}\text { TIPO DE } \\
\text { TURISTA }\end{array}$} & \multicolumn{10}{|c|}{ ACTIVIDADES DE PREFERENCIA PROVINCIA DE CHIMBORAZO } \\
\hline & Tren & $\begin{array}{l}\text { Visitar } \\
\text { iglesias }\end{array}$ & $\begin{array}{l}\text { Visitar } \\
\text { museos }\end{array}$ & $\begin{array}{l}\text { Excursión } \\
\text { Chimborazo }\end{array}$ & $\begin{array}{c}\text { Visitar } \\
\text { comunidades }\end{array}$ & $\begin{array}{c}\text { Visitar } \\
\text { mercados }\end{array}$ & $\begin{array}{c}\text { Compra de } \\
\text { productos y } \\
\text { artesanías }\end{array}$ & Negocios & $\begin{array}{l}\text { Visitar } \\
\text { parques }\end{array}$ & Total \\
\hline \multirow{2}{*}{$\begin{array}{l}\text { Turista } \\
\text { nacional }\end{array}$} & 64 & 55 & 26 & 67 & 29 & 39 & 26 & 3 & 13 & 321 \\
\hline & $20 \%$ & $17 \%$ & $8 \%$ & $21 \%$ & $9 \%$ & $12 \%$ & $8 \%$ & $1 \%$ & $4 \%$ & $100 \%$ \\
\hline \multirow{2}{*}{$\begin{array}{l}\text { Turista } \\
\text { extranjero }\end{array}$} & 14 & 9 & 4 & 13 & 7 & 7 & 4 & 1 & 2 & 61 \\
\hline & $23 \%$ & $14 \%$ & $7 \%$ & $21 \%$ & $12 \%$ & $11 \%$ & $7 \%$ & $2 \%$ & $3 \%$ & $100 \%$ \\
\hline \multirow{2}{*}{ TOTAL } & 78 & 63 & 30 & 80 & 36 & 45 & 30 & 4 & 15 & 382 \\
\hline & $20 \%$ & $17 \%$ & $8 \%$ & $21 \%$ & $9 \%$ & $12 \%$ & $8 \%$ & $1 \%$ & $4 \%$ & $100 \%$ \\
\hline
\end{tabular}

Fuente: Elaboración propia.

Actualmente, el internet es la principal fuente de información tanto para turistas nacionales como extranjeros, es así que de la encuesta realizada para obtener información referente a la forma de cómo han llegado a conocer los destinos turísticos de la ciudad de Riobamba y la provincia de Chimborazo, se obtuvieron los resultados mostrados a continuación en la tabla 4.

Tabla 5: Principal fuente de información de los turistas.

PRINCIPAL FUENTE DE INFORMACIÓN

\begin{tabular}{|c|c|c|c|c|c|c|c|c|}
\hline TIPO DE TURISTA & RADIO & TV & INTERNET & $\begin{array}{c}\text { GUÍA } \\
\text { TURÍSTICA }\end{array}$ & FAMILIAR & AMIGOS & $\begin{array}{c}\text { AGENCIA } \\
\text { VIAJES }\end{array}$ & Total \\
\hline \multirow{2}{*}{ Turista nacional } & 10 & 45 & 125 & 13 & 55 & 55 & 19 & 321 \\
\hline & $3 \%$ & $14 \%$ & $39 \%$ & $4 \%$ & $17 \%$ & $17 \%$ & $6 \%$ & $100,00 \%$ \\
\hline \multirow{2}{*}{ Turista extranjero } & 1 & 3 & 31 & 11 & 2 & 7 & 5 & 61 \\
\hline & $1 \%$ & $5 \%$ & $50 \%$ & $18 \%$ & $4 \%$ & $12 \%$ & $9 \%$ & $100,00 \%$ \\
\hline \multirow{2}{*}{ TOTAL } & 10 & 48 & 156 & 24 & 57 & 62 & 25 & 382 \\
\hline & $3 \%$ & $13 \%$ & $41 \%$ & $6 \%$ & $15 \%$ & $16 \%$ & $6 \%$ & $100 \%$ \\
\hline
\end{tabular}

Fuente: Elaboración propia.

\section{Conclusiones.}

Una vez realizada la investigación cuantitativa a turistas nacionales y extranjeros; y la investigación cualitativa a informantes calificados, gestores culturales y responsables de atractivos turísticos en la ciudad de Riobamba, se identificó la necesidad de emprender un programa de logística para de la difusión de frecuencias de transporte interprovincial, interprovincial, intercantonal e interparroquial, pues como bien lo afirma Ninoska Viloria (11) "de nada sirve tener instalaciones, servicios, infraestructuras si no hay medios para desplazarse y estar en el sitio deseado". Es importante que los turistas se encuentren informados de los horarios, rutas, tarifas, debido a que el transporte es un factor determinante del producto turístico de una ciudad, por ello, todos los integrantes básicos del 
sector turístico, tales como: alojamiento, centros de atractivos turísticos, agencias de viajes, deben coadyuvar a mejorar la cadena de valor de la actividad turística de la provincia de Chimborazo.

Diseñar paquetes comerciales orientados a turistas nacionales, internacionales e intermediarios del sector turístico, en el cual se incluya información de horario, tarifas y destinos de las diferencies frecuencias de transporte provincial. En estos productos, se establecerán los lugares turísticos de la ciudad y actividades culturales, gastronómicas, que incluyan 203 noches de duración. Es importante, apoyar la campaña promocional en instituciones vinculadas al sector turístico, además, impulsar continuamente eventos culturales novedosos, para que la ciudad tenga reconcomiendo en el mercado turístico, y a su vez enriquecer la oferta cultural.

Diseñar una campaña publicitaria en internet y redes sociales, en la que se resalten y promocionen los atributos de los atractivos turísticos de la provincia de Chimborazo, incluyendo información e historia de los mismos, considerando que acuerdo con el estudio cuantitativo, la principal fuente de información tanto para turistas nacionales como extranjeros es el internet. Adicionalmente se debe considerar que, para los turistas extranjeros, la segunda fuente de información son las guías turísticas, por ello, es importante pautar en este medio publicitario, específicamente en las guías turísticas europeas, ya que es de este continente de donde proviene la mayoría de los visitantes.

Abrir una oficina con punto de información en cada una de las terminales terrestres del cantón Riobamba, como son la interprovincial, intercantonal e interparroquial a fin de brinda una información adecuada a los turistas sobre los nuevos productos que se encuentren ofertando y las distintas alternativas de frecuencias de transporte a sus diferentes destinos.

La ciudad cuenta con varias operadoras de transporte terrestre debidamente autorizadas, debiendo canalizar a través de estas los flujos de turistas hacia otros destinos turísticos, considerando que el $37 \%$ de turistas utiliza este transporte, por ello, se hace de imperiosa necesidad elaborar un plan de logística del transporte en la provincia de Chimborazo a fin de fortalecer la organización de las frecuencias de transporte en sus diferentes clases y ámbitos para proveer a los turistas de un servicio directo a su lugar de destino, garantizándoles en todo momento seguridad y confort en sus viajes; para ello se deberá elaborar un distributivo de cada una de las operadoras de transporte terrestre, las unidades con las que se cuentan y los horarios fijos que deben cumplir todos los días del año para el traslado de turistas a los principales destinos turísticos de la provincia de Chimborazo, como son: Alausí para realizar la ruta del Tren (Alausí - Nariz del Diablo) y nevado Chimborazo. 


\section{Referencias bibliográficas.}

1. Ministerio de Turismo del Ecuador. MINTUR (2014). Proyecto Ecuador Potencia Turística.

2. Tamayo, D. (2012). Plan de promoción y difusión turística para la parroquia San Juan de Pastocalle, ubicado en el cantón Latacunga, provincia de Cotopaxi. Obtenido de: http://repositorio.utc.edu.ec/handle/27000/1570.

3.Organización Mundial de Turismo (2012) UNWTO. Obtenido de: http://www.eunwto.org/doi/pdf/10.18111/9789284414680

4. Muñoz, A. (2005). LOGÍSTICA Y TURISMO. MADRID, ESPAÑA.: Ediciones Díaz Santos S.A.

5. Cabeza R., M. (2001). LA LOGÍSTICA EN LA ACTIVIDAD TURÍSTICA. Revista Venezolana de Análisis de Coyuntura, VII (2), 257-264.

6. Turismo, M. d. (2015). PROYECTO ECUADOR POTENCIA TURÍSTICA. Obtenido de https://www.turismo.gob.ec/wp-content/uploads/2015/04/Documento-Proyecto-Ecuador-PotenciaTur\%C3\%ADstica.pdf

7. Asamblea Nacional del Ecuador. (2008). LEY ORGÁNICA DE TRÁNSITO, TRANSPORTE TERRESTRE Y SEGURIDAD VIAl. Corporación de Estudios y Publicaciones. Quito

8. Asamblea Nacional del Ecuador. (2016). REGLAMENTO A LA LEY ORGÁNICA DE TRÁNSITO, TRANSPORTE TERRESTRE Y SEGURIDAD VIAL. Corporación de Estudios y Publicaciones. Quito

9. (González Alpuche, 1978: 564)

10. Asamblea Constituyente del Ecuador. (2008). CONSTITUCIÓN DE LA REPÚBLICA DEL ECUADOR. Corporación de Estudios y Publicaciones. Montecristi. Ecuador

11. Viloria, N. (2012). DESARROLLO TURÍSTICO Y SU RELACIÓN CON EL TRANSPORTE. Obtenido de: Universidad Experimental Nacional Libertador: http://mingaonline.uach.cl/pdf/gestur/n17/art02.pdf

12. Lundberg, D. (1977). El negocio del turismo, México.

13. De la Torre Padilla O.(1980). El turismo. Fenómeno Social.

14. Ricaurte, C. (2007). MARKETING TURÍSTICO. Facultad de Recursos Naturales Escuela de Ecoturismo Escuela Superior Politécnica de Chimborazo. Riobamba.

15. PLADETUR 2020.

16. Plan estratégico institucional del GADM Riobamba 2017. 
Bertoncello, R. (2002). Turismo, territorio, otras prácticas, otras miradas. Obtenido de www.nulan.mdp.edu.ar

Cabeza R., M. (2000). La industria turística busca nuevos horizontes. Economía, XXV (16), 33-44.

Canavos, G. (1988). Probabilidad y Estadística. Aplicaciones y Métodos. Mc Graw Hill Interamericana de México.

Council, W. T. (2016). Travel \& Tourism. Economic Impact 2016 World. Obtenido de: https://www.wttc.org//media/files/reports/economic\%20impact\%20research/regions\%202016/world2016.pdf

Dirección de Gestión de Turismo - Municipio Riobamba. (2016). Atractivos turísticos. Riobamba.

Guiltinan, Joseph, Gordon Paul, Thomas Madden. (1998). Gerencia de Marketing Estrategias y programas . Colombia: Mc Graw Hil.

Foro Turismo, 2011. Obtenido de: https://www.tecnohotelnews.com/2011/10/la-importancia-deltransporte-en-el-sector-turistico.

Herrera, Maria. Plan estratégico para el desarrollo sustentable del turismo en la ciudad de Riobamba, provincia de Chimborazo. Obtenido de: http://dspace.espoch.edu.ec/handle/123456789/8318.

Instituto Nacional de Estadística y Censos. (2010). Censo de Población y Vivienda . Obtenido de: http://www.ecuadorencifras.gob.ec/base-de-datos-censo-de-poblacion-y-vivienda-2010.

Instituto Nacional de Estadística y Censos. (2010-2020). Proyección población ecuatoriana, por años calendario, según cantones. Obtenido de: www.ecuadorencifras.gob.ec

Macedo, B. (2005). El concepto de sostenibilidad. Organización de las Naciones Unidas para la Educación, la Ciencia y la Cultura. Oficina Regional de Educación para América Latina y El Caribe . Santiago: UNESCO.

Cedeño, N. E. V. (2012). Desarrollo turístico y su relación con el transporte. Gestión turística, 1(17), 23-36.

Malhotra, N. (2004). Investigación de mercados. México: Pearson Prentice Hal, Cuarta edición.

Milla, D. M. (2012). La elaboración del plan estratégico y su implementación a través del cuadro de mando integral. Madrid, España.: Ediciones Díaz Santos S.A.

OMT. (2016). Organización Mundial de Turismo. Recuperado el 2016, de https://www.eunwto.org/doi/pdf/10.18111/9789284418152

Planificación, C. N. (2013 - 2017). Plan Nacional del Buen Vivir . Obtenido de: http://www.buenvivir.gob.ec/descarga-objetivo 
Pirages, D. (1977). The Sustainable society: implications for limited growth. Editorial: Nueva York, Praeger. Estados Unidos.

Quesada, R. (2005). Elementos del turismo . San José, Costa Rica: Editorial Universidad Estatal a Distancia.

Riobamba, D. d. (2016). Boletín Estadísticas Turísticas. Riobamba.

Riobamba, I. M. (2002). Guía práctica para el turista. Riobamba.

SENPLADES. (2013). Secretaría Nacional de Planificación y Desarrollo . Recuperado el 2016

SEGITTUR (Sociedad Estatal para la Gestión de la Innovación y las Tecnologías Turísticas), 2011. Recuperado el 6 enero de 2019 de, https://www.tecnohotelnews.com/2011/10/la-importancia-deltransporte-en-el-sector-turistico.

Xercavins, J., Cayuela, D., Cervantes, G., Sabater, A.. (2005). Desarrollo sostenible. Barcelona, España. 80 - 82. 


\section{PARA CITAR EL ARTÍCULO INDEXADO.}

Herrera Chico, M., Arguello, D., Villamarín Padilla, J., \& Guerra Sarche, X. (2019). Escasa difusión de frecuencias de transporte turístico y logística, en la ciudad de Riobamba, análisis legal, matemático - estadístico. Ciencia Digital,3(2.2), 102-117. https://doi.org/10.33262/cienciadigital.v3i2.2.463

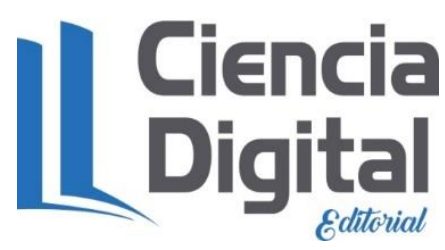

El artículo que se publica es de exclusiva responsabilidad de los autores y no necesariamente reflejan el pensamiento de la Revista Ciencia Digital.

El artículo queda en propiedad de la revista y, por tanto, su publicación parcial y/o total en otro medio tiene que ser autorizado por el director de la Revista Ciencia Digital.
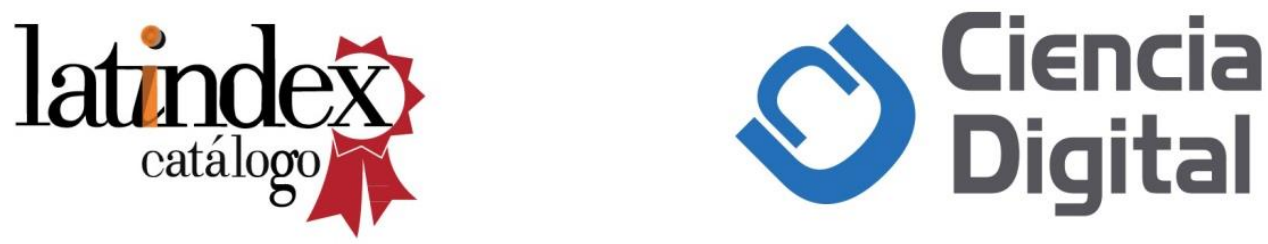